\title{
EL MÉTODO COMPARADO COMO COMPONENTE METODOLÓGICO EN LAS POLÍTICAS PÚBLICAS ${ }^{1}$
}

\section{THE COMPARATIVE METHOD AS A METHODOLOGICAL COMPONENT IN PUBLIC POLICIES}

\author{
Mijael Altamirano-Santiago ${ }^{2}$ (D); Abigail Martínez-Mendoza ${ }^{3}$ (iD). \\ 2. Centro de Investigaciones Económicas, Administrativas y Sociales del Instituto Politécnico Nacional, México. \\ maltamiranos@ipn.mx \\ 3. Universidad Autónoma Metropolitana, Unidad Lerma, Departamento de Procesos Sociales, México. \\ a.martinez@correo.ler.uam.mx. \\ *Correspondencia del Autor: Mijael Altamirano-Santiago, correo electrónico: maltamiranos@ipn.mx.
}

\section{RESUMEN}

Objetivo. Exponer de la valía del método comparado como elemento base para la gestación, elaboración y ejecución de políticas públicas en las diversas esferas de la acción gubernamental. Método. Detallar el significado del método comparado y su uso como principal herramienta en los trabajos académicos (o de investigación) que se adscriben en la subdisciplina de las políticas públicas. Resultado. Se desataca que en los diferentes ámbitos de las políticas públicas no es limitativo el uso del método comparado. Conclusión. Se observa que el estudio de casos —una de las vertientes del método comparado - tiene mayor uso en los trabajos académicos (o de investigación); contrario, a lo que acontece en la formación de los educandos en donde el método comparado en sus vertientes (el estudio de casos y el estudio de caso) cobran relevancia en el proceso de enseñanza-aprendizaje.

Palabras clave: Políticas públicas; método comparado; estudio de casos; estudio de un solo caso.

Cómo citar:

Altamirano-Santiago, Mijael; Martínez-Mendoza, Abigail. (2020). El método comparado como componente metodológico en las políticas públicas. Revista de Investigaciones Universidad del Quindio, 32(2), 88-101. https://doi.org/10.33975/riuq.vol32n2.455

Información del artículo: Recibido: 15 noviembre 2020; Aceptado: 28 diciembre 2020

1 Esta es una versión revisada y actualizada de Altamirano y Martínez (2011). "El método comparado y el neo-institucionalismo como marco metodológico para la investigación en las Ciencias Sociales", Mundo Siglo XXI, CIECAS-IPN, núm. 25, vol. VII, pp. 55-63. 


\begin{abstract}
Objective. To expose the value of the comparative method as a base element for gestation, development and implementation of public policies in the various areas of government action. Method. Explain the meaning of the comparative method and its use as the main tool in academic (or research) works that are assigned to the subdiscipline of public policies. Result. It should be noted that the use of the comparative method is not limiting in the different areas of public policy. Conclusion. It is observed that case studies — one of the aspects of the comparative method- is more widely used in academic (or research) works; contrary to what happens in the formation of learners where the comparative method in its aspects (the case studies and case study) become relevant in the process of teaching and learning.
\end{abstract}

Keywords: public policies, comparative method, case studies, case study.

\section{INTRODUCCIÓN}

Sin duda alguna prevalecen un sinfín de métodos, teorías o técnicas para dar cuenta de la realidad social y/o de los fenómenos que acontecen en cualquier unidad de análisis como objeto-sujeto de estudio. Por lo consiguiente, no existe hasta ahora realidad social o unidad de análisis alguna sin que su tratamiento sea explicado con rigor científico. De ahí que, se afirme que siempre habrá un referente metodológico, teórico o técnico que exponga con solidez el objeto-sujeto de estudio.

De todas las herramientas conceptuales y procedimentales que emanan de las teorías y/o métodos, cobra especial relevancia el método comparado como marco metodológico por agregar valor y rigor científico al tratamiento y entendimiento de la realidad social y/o de los fenómenos que acontecen en cualquier unidad de análisis como objeto-sujeto de estudio, sea a través del estudio de casos o el case study.

Esto es así, si el objeto-sujeto de estudio es una unidad de análisis o realidad social definida o delimitada por su propia caracterización. No así en lo referente a las políticas públicas que éstas emergen en un contexto más amplio y completo por su propio axioma, lo que en consecuencia complejiza su estudio y entendimiento y, aún, si únicamente se realizan estudios descriptivos o monográficos.

Por lo que, pasar de las descripciones a las explicaciones, el método comparado muestra su valía como una herramienta de gran utilidad, no sólo para quien(es) está(n) en el proceso formativo en la subdisciplina de las políticas públicas, sino también para quien(es) ya ha(n) consolidado su trayectoria como investigador, pues permite desarrollar y ampliar las habilidades de observación, tan necesarias para la investigación, incluso para incrementar las capacidades de abstracción y análisis a partir de los casos de estudio.

\section{MARCO CONCEPTUAL}

\section{Método comparado}

El método comparado, también llamado comparativismo, es una herramienta metodológica que agrega valor a los estudios y análisis en el ámbito de las ciencias sociales, para construir discursos congruentes y de alto rigor respecto a una realidad social. El método comparado permite contar con un mayor número de elementos que apoyan para comprender la especificidad de una situación, toda vez que el método explicita las diferencias y similitudes en el origen y desarrollo de los fenómenos sociales en contextos diversos y/o similares al del caso que se estudia (Mackie y Marsh, 1997; Blondel, 
1999).

Para una mayor comprensión del método comparado, es importante diferenciar entre metodología y marco metodológico. Se trata de acepciones estrechas, pero diferentes, tal como se expone a continuación:

i. La metodología es de mayor estatus $\mathrm{y}$ alcance por constituirse racional y sistemáticamente como un conjunto de operaciones y procedimientos con miras a encontrar soluciones óptimas a problemas complejos, teóricos o prácticos; incluso es universal por estar presente en todos los campos de conocimiento (Morales, 2002; Altamirano y Martínez, 2011).

ii. El marco metodológico es una sucesión de acciones particulares y concretas en el tratamiento del objeto-sujeto de estudio; y que derivado de un ejercicio arduo de análisis, facilita un procedimiento ordenado, pasando por la selección del objeto-sujeto de estudio, la exposición de objetivos, la articulación de la justificación y definición de la problematización (o planteamiento del problema o estado de la cuestión), así como la formulación de hipótesis, selección del método, teoría o técnica para la comprobación de hipótesis $\mathrm{y}$, finalmente, la formulación del discurso o de la narrativa sobre el fenómeno observado y/o tratado (Morales, 2002; Altamirano y Martínez, 2011).

Así, la metodología es una acepción amplia tendiente a las reflexiones epistemológicas, y el marco metodológico es una acepción restringida, con un sentido más instrumental y operacional (Silva, Martínez, Altamirano y Lara, 2020). Anotada esta diferencia, es posible afirmar que el método comparado se inscribe sobre la línea de los marcos metodológicos, por su orientación instrumental del procedimiento sucesivo de acciones encaminadas al estudio de un objetosujeto.
El método comparado implica necesariamente observar las diferencias y similitudes que se dan al "confrontar una cosa con otra" (Sartori, 1994, p. 31). Si los objetos de estudio están alejados del referente de similitud, es decir, son totalmente diferentes, se invalida la cláusula ceteris paribus del método comparado como lo refiere el propio Sartori (1994, p. 40), si es así el estudio y análisis se complican porque no se distinguen analogías existentes entre los casos observados (Altamirano y Martínez, 2011).

Siendo así, la comparación tiene cabida científica cuando se trata de un acto consciente que tiene presente el objetivo de la investigación y procede en el análisis de semejanzas y diferencias, pues la comparación supone, al mismo tiempo, su existencia (Mackie y Marsh, 1997; Blondel, 1999). Aunado a esto, Mèny y Thoënig (1992) afirman que los trabajos de investigación resultan más interesantes y de mayores alcances por la superposición de resultados, haciendo del comparativismo un método en donde prevalecen explicaciones manifiestas y no difusas, subyacentes y no endebles. A esto, Collier (1994) señala que este método resulta ser un estímulo para la formulación de conceptos porque incita las capacidades analíticas, incluso promueve habilidades de observación. El acto consciente de observar constituye un método de comprobación en las ciencias sociales, en tanto su principio es la consciencia y la sistematicidad; lo que vale para la observación, vale para la experimentación, en etapas distintas, según Bourdieu (1975). A todo esto, existen diferentes apreciaciones que no cuestionan la razón del método comparado, pero exponen matices diferentes a considerar.

De acuerdo con Wiarda (1991) el método comparado conduce a que las explicaciones se alejen del etnocentrismo o localismos, en donde se asienta el fenómeno observado. Por lo que Przeworski (1987), señala que el valor del método comparado es explicar los fenómenos y las realidades en estadios diversos para, así, propiciar la generación de una teoría o la formulación de conceptos. O bien, como apunta 
Macridis (1981), la carencia de la comparación genera explicaciones esencialmente descriptivas, localistas y monográficas.

En el caso de Rose (1991), si el método comparado refiere al ámbito local, se debe considerar el tiempo en un período determinado para observar y determinar un pasado y un presente, de tal forma que los estudios sean diacrónicos y/o sincrónicos. Así, el método comparado permite tener presente las diferencias y similitudes en el origen $\mathrm{y}$ desarrollo de la unidad de análisis, en contextos distintos y/o similares; contar con elementos que apoyen a comprender la especificidad o similitud de cada unidad de análisis o caso (Altamirano y Martínez, 2011).

Blondel (1999) señala que el método comparado es una herramienta que examina dos o más realidades; a esto, se debe agregar que estas realidades deben necesariamente guardar cierta analogía (o cierta similitud), pues de lo contrario se dificultaría la comparación (Sartori, 1994).

\section{Tipología del método comparado: Estudio de casos y case study}

Para Borges (1995), el estudio de los casos se vuelve relevante por la exigencia del análisis, por no limitarse a un enfoque o área de estudio, lo que en consecuencia favorece a la interdisciplinaridad/ multidisciplinaridad al describir y explicar los fenómenos que acontecen ámbitos diversos $\mathrm{y}$ complejos, sean actores específicos, individuales institucionales $\mathrm{u}$ organizacionales.

De acuerdo con Merriam (1988), el método comparado permite abordar unidades sociales complejas, donde las múltiples variables son de vital importancia para la 'comprensión' del fenómeno como lo denomina Creswell (2002). Paralelamente, Collier (1994) indica que el estudio de casos es útil para generar hipótesis y confirmar o descartar una teoría; esta categoría permite hacer un examen intensivo de casos, siempre y cuando el número casos tomado para el estudio sea reducido (Altamirano y Martínez,
2011).

El estudio de casos plantea dos esquemas para el tratamiento del objeto-sujeto de investigación o de la unidad de análisis: i) el estudio de caso (case study) — también llamado estudio de un solo caso-; ii) el estudio de pocos casos con muchas variables, en donde el objetivo es encontrar la relación entre variables para, así, construir teorías o proposiciones; $y$, iii) el análisis de pocas variables con muchos casos, tiene como propósito hallar el vínculo entre los casos $\mathrm{y}$, en consecuencia, dar cuenta de las diferencias o similitudes existentes que posibiliten explicar tal relación a efecto de generar axiomas más acabadas.

El estudio de caso, si bien podría estar lejano de lo que en esencia representa el método comparado, tal afirmación no es exacta. Esta tesis va en el sentido en que el case study se inscribe en un plano localista, individual, descriptivo, monográfico, etnocentrista (Macridis, 1981; Wiarda,1991), lo que en consecuencia podría ser una debilidad al tener el periodo de tiempo (sincrónico/diacrónico) como base para revelar similitudes y diferencias en una unidad de análisis (Altamirano y Martínez, 2011). Al tiempo, también, permite un examen intensivo, aunque con recursos limitados y con aportaciones débiles. Para Llamazares (1995) y Collier (1994), el estudio de un caso no permitiría avanzar más allá de la naturaleza localista del acontecimiento.

Por el contrario, el estudio de pocos casos permite un adecuado manejo de hipótesis (Caïs, 1997; Lijphart, 1971; Collier, 1994), así como también permite el control de la investigación y facilita observar similitudes y diferencias. El nivel de abstracción es menor en el case study que en el estudio de pocos casos (Altamirano y Martínez, 2011).

Caïs (1997) indica que al comparar un número amplio de casos es difícil analizar todas las similitudes y diferencias, ello reduce la probabilidad de encontrar uniformidad entre 
casos, de tal forma que se desborda el análisis sobre el objeto-sujeto de investigación (Lijphart, 1971). Sin embargo, para Mèny y Thoënig (1992) el estudio de un número amplio de variables (o casos) permite construir una tipología de estos; tal como construir el estado del arte en determinada materia; que bien podría señalarse como el primer momento del estudio comparado, para después determinar cuáles casos serán incluidos o excluidos del análisis.

A todo esto, de acuerdo con Blondel (1999), la literatura existente no señala puntualmente el número exacto de casos a tomar en cuenta para un análisis comparado. Esto es entendible ya que los casos, por su propia naturaleza, se caracterizan por las condiciones en las que tienen lugar, y eso dificulta afirmar para cuáles circunstancias se debe analizar determinado número de casos. De este modo, puede decirse que el número de casos estará en función del objetivo de la comparación, ya sea: 1) determinar las semejanzas y diferencias a través de establecer una relación entre las variables; es el escenario de pocos casos y muchas variables; o bien, 2) determinar el vínculo entre los casos, es el escenario de muchos casos y pocas variables. A fin de cuentas, es destacar las diferencias o similitudes, ya sean generales o concluyentes.

El estudio de un número limitado de casos viene a ofrecer un análisis profundo de cada una de las variables, fenómenos o hechos; a facilitar las proposiciones, y hacer observable las diferencias y similitudes de cada uno de los casos (Altamirano y Martínez, 2011).

\section{Limites del método comparado}

Hasta aquí se pueden observar las virtudes del método comparado para el estudio de diferentes unidades de análisis. Mèny y Thoënig (1992), así como Lijphart (1971), señalan, que el método comparado presenta debilidades para confrontar y controlar las hipótesis cuando se contemplan muchas variables y pocos casos, sea para formular teorías, proposiciones o axiomas.
Es decir, el riesgo se presenta cuando el número de variables es inmanejable por su capacidad limitada para manifestarse en pocos casos de estudio; y lo mismo en sentido opuesto, muchos casos y pocas variables resultará en descripciones monográficas y no comparativas.

Una forma de contener este riesgo metodológico, donde se presentan muchas variables, es: a) fijar el estudio sobre variables específicos o particulares; b) reducir los casos de las unidades de análisis; c) combinar una o dos variables que expresen similitud o diferencia; d) incrementar el número de casos de manera moderada; e) hacer comparaciones sistemáticas con un número limitado de casos; y f) elegir casos afines, similares o idénticos, salvo en el factor que se desea analizar (Rose, 1991; Wright, Hague, Harrop y Breslin, 1992; Mackie y Marsh, 1997; Lijphart, 1971).

Por todo ello, resulta necesario determinar el objeto-sujeto de estudio, el registro detallado y crítico de las observaciones recabadas, el objetivo de comparar para, finalmente, analizar e interpretar los registros en función de un marco teórico referencial (Álvares-Gayou, 2006).

\section{Las políticas públicas: significado y alcance}

Si se revisa la literatura sobre la bibliografía existente acerca de las políticas públicas es posible saber que existe un vasto número de adjetivos sobre esta subdisciplina, si se considera que responde a las necesidades sociales que el Estado debe satisfacer. Ejemplo de ello, es cuando se habla desde el gobierno de las acciones públicas que se desarrollan en sus diversas áreas administrativas: política educativa, política social, política científica y tecnológica, política agraria, política fiscal, y un largo etcétera. Bajo este paraguas que impone la acción pública, se complejiza determinar un concepto absoluto o concluyente, toda vez que tal acción responde a una necesidad y ésta depende del contexto en donde se origina y a las condiciones que emergen en su en torno. 
De este modo, no sólo es limitado afirmar que las políticas públicas son cursos de acción o acciones de gobierno (Aguilar, 1992), sino que también reduce en gran medida su importancia en la ciencia política y en el entramado las distintas áreas disciplinares. Por lo que diversos autores dan cuenta de la prevalencia de conceptualizaciones, variantes y componentes de las políticas públicas. Así, Dye (1975) indica que una política pública es aquello que una autoridad decide hacer o no hacer. Para Larrue (citado por Kauffer, 2002), afirma que las políticas públicas tratan de una sucesión de decisiones, actividades o de medidas coherentes tomadas principalmente por los actores del sistema político-administrativo a fin de resolver un problema colectivo. Esto, de acuerdo con Kauffer (2002), se debe formalizar a través de mecanismos coercitivos para moldear los comportamientos de los grupos objetivo. Es decir, las acciones sucesivas para atender un problema de la sociedad deben contemplar determinados dispositivos o componentes conducentes o próximos al resultado deseado.

Por su parte, Lahera (2004) señala que las políticas públicas serán aquellas soluciones específicas de determinados asuntos públicos $\mathrm{y}$, que, en éstas, deberían participar el poder público y los diversos sectores (sean sociales, productivos, empresariales, económicos o privados, entre otros). Para Oszlak y O’Donnell (1995) las políticas públicas constituyen respuestas en función de las condiciones del problema, aunque éstas no deben ser vistas como una respuesta a una demanda específica.

Mèny y Thoënig (1992) indican que una política pública se formula como un programa de acción gubernamental para atender a un sector de la sociedad o en un espacio geográfico. En especial, Thoënig (2006) afirma que las políticas describen o demandan tres condiciones: i) la condición deseada, ii) la condición presente, y iii) la condición futura.

Otra acepción es la que propone Aguilar (1992), quien anota que las políticas públicas son un conjunto de cursos de acción, por un lado, y, por el otro, son cursos deliberadamente diseñados para responder a una necesidad social, sin que se especifique que este conjunto de cursos de acción debiera ser el resultado de la acción colectiva (Lahera, 2004) y que según el neoinstitucionalismo debe ser así, al considerar el en torno como eje para su gestación, elaboración e implementación (Altamirano y Martínez, 2011).

De este número de conceptualizaciones, sobresalen: 1) la interacción de actores; 2) las condiciones prevalecientes en las que tienen lugar las políticas públicas; y 3) las condiciones deseadas. Por lo que, es posible señalar que, según las condiciones (o condicionantes) y/o necesidades serán los cursos de acción que habrán de apuntalarse y los actores que habrán de involucrarse.

Mención aparte merece referir a las políticas públicas desde el enfoque de los paradigmas (Khun, 1971) ${ }^{1}$ que, dicho sea de paso, se podría afirmar que en el paradigma clásico, las políticas públicas se implementaron en el marco del Estado de Bienestar (también llamado estado benefactor, estado providencia o sociedad del bienestar) porque hubo énfasis en la protección a los trabajadores asalariados, así como la consolidación de la naciente clase media y su capacidad de compra de los bienes nacionales (González, 2000; Farge, 2007).

Hoy día, con la llegada del estado neoliberal se transforma el paradigma de antaño para dar vigencia a un nuevo paradigma (liberal, en este caso) según González (2000) y, por consiguiente, cambia los referentes del Estado de Bienestar. En efecto esto es así, si se tiene presente que en el centro del Estado neoliberal hay un predominio en el actuar de los agentes del Estado y de los sectores sociales: el libre mercado, la globalización, liberalización de

1 T. Khun señala que la evolución de las ciencias se produce a través de crisis y rupturas epistemológicas, que denomina revoluciones, las cuales dan lugar a nuevas concepciones, planteamientos teóricos y, en algún momento determinado, paradigmas, que vuelven a la crisis. 
la economía, entre otros. El estado neoliberal según David Harvey (citado por Cárdenas, 2017) “(...) es una teoría geopolítica de dominación y no sólo es una estructura económica, sino un esquema integral que conjuga la violencia política, militar, ideológica, jurídica y estatal, para que las transformaciones estructurales que promueve a nivel nacional y global, pongan a las anteriores variables de su lado con el propósito de modificar, en beneficio de las clases dominantes, los elementos que conforman la convivencia social de la nueva forma de dominación política" (p. 168).

Aunado a lo anterior, también es pertinente afirmar que de este modelo, se gesta un paradigma neoliberal en donde cada vez más se observan mecanismos de gobernanza, gobernabilidad, legitimidad, legalidad y, sobre todo, un nuevo proceso de toma de decisiones apegado a los dictados del neoinstitucionalismo (Altamirano y Martínez, 2011) que favorecen la participación y corresponsabilidad de los diferentes actores (sociales, productivos, económicos, entre otros) adscritos en el régimen político (March y Olsen, 1984; North,1993; Koelble, 1995; Hall y Taylor, 1998; Camou, 2001).

De estos rasgos que impone el nuevo paradigma, la que cobra mayor relevancia es la gobernanza en las políticas públicas, con sus respectivos matices según la materia correspondiente, lo que en consecuencia induce que los instrumentos también se diversifiquen y se especialicen. De ahí que, las políticas públicas deban materializarse en términos jurídicos e institucionales a través de sus propios instrumentos, en tanto que éstos contienen ordenamientos jurídicos que expresan reglas, principios y valores con miras a regular: el poder, las relaciones con los ciudadanos y la garantía de los derechos (DEJ-RAE, 2017). En este sentido, los instrumentos conducen a la concreción de las políticas públicas al quedar señalado de manera explícita la asignación de recursos, sean éstos económicos, financieros, físicos, técnicos o humanos (Fernández, Bello y Messarini, 2016).
¿Y cuáles son los instrumentos de política pública? Éstos, son de tres tipos: i) Instrumentos normativos, los que estructuran y orientan las actividades; ii) Instrumentos de gestión, los que facilitan la concreción de actividades; y iii) Instrumentos de recursos, los que hacen viable las acciones (Isuani; 2012). Por ello, es menester señalar que la instrumentación y/o ejecución de las políticas públicas es compleja y diversa, dado que responden a necesidades, demandas $\mathrm{y}$ afectaciones (sean políticas, sociales $\mathrm{y} / \mathrm{o}$ económicas).

La instrumentación de políticas públicas por sí misma no es una etapa que forme parte del círculo del diseño "problema-agenda-gestaciónelaboración-implementación-evaluación” de la acción de los poderes públicos, más bien, se erige como una actividad trasversal en todo el círculo. Por lo consiguiente, las políticas públicas están lejos de ser una tarea incierta o arriesgada cuando éstas se construyen bajo la visión de Estado, al responder a una tarea que deriva de un conjunto de factores, necesidades o circunstancias presentes en la realidad social, que dicho sea de paso, privilegia al interés colectivo. Situación contraria, ocurre cuando éstas son formuladas bajo una visión ideológica (o de gobierno), al responder a un interés particular a efecto de cobrar visibilidad en el entramado social.

Lo anterior puede ejemplificar la conceptualización de políticas públicas, aún con la diversidad y complejidad para ofertar un concepto definitivo (o exhaustivo). En lo que toca, a las conceptualizaciones sobre políticas públicas en materia social cambian con el paso del tiempo (verbi gratia, las nuevas categorías para definir los componentes de pobreza y su caracterización) y de las condiciones subyacentes.

En efecto es así, si se tiene presente a Catalá (1951) cuando afirma que la política social desde la perspectiva económica - es una acción de gobierno para subsanar las desigualdades económicas derivadas del proceso distributivo. Al tiempo también, refiere que -desde la 
perspectiva sociológica-, las políticas sociales son las medidas sistemáticas para regular las relaciones entre las clases respecto a los poderes públicos y la equidad. De ahí que, la política social —según Catalá- aspira a resolver los problemas a través de la justicia social. Se debe resaltar que el autor refiere diferentes ángulos desde los cuales se pueden abordar las políticas sociales (1951).

Fernández y Rozas (1988), por su parte, señalan que las políticas sociales tratan del conjunto de decisiones que implica determinados derechos y obligaciones. Más reciente, Atria (2005) indica que las políticas sociales son la articulación de acciones, mecanismos e instrumentos, conducidos por un agente público, cuyo objetivo es mejorar las oportunidades de ciertos grupos sociales. En cambio, Ortíz (2007) menciona que las políticas sociales se configuran como servicio o asistencia social, sea de educación, de salud o de seguridad social, al tiempo, también, señala que las políticas sociales contemplan la distribución, protección y justicia social, y la incorporación de los ciudadanos al núcleo de las políticas públicas. Por su parte, De la Torre, Rodríguez-Oreggia y Soloaga (2018) explican que las políticas sociales son las acciones públicas dirigidas a proteger y promover el desarrollo y el gasto social para la educación, salud, seguridad social, vivienda $\mathrm{y}$ asistencia social.

Estas acepciones puestas en evidencia, permiten observar que las conceptualizaciones responden al momento histórico y a la coyuntura en la que tienen lugar, sin que se ponga de manifiesto diferencia significativa alguna en el alcance del concepto de las políticas sociales. Lo real es que las condiciones de una definición u otra son reflejo del paradigma predominante, y frente a la descripción de las políticas públicas, es necesario contar con referentes tipológicos que permitan mayor comprensión de la dirección que pueden tomar la acción de los poderes públicos.

Así, es posible identificar que en las conceptualizaciones de las políticas públicas, destacan, que éstas: i) responden a una vasta diversidad de materias/necesidades; ii) sus objetivos están sujetos a las condiciones prevalecientes en el que tienen lugar; iii) aspiran a responder a necesidades consensuadas con los actores involucrados; iv) son patentes en la medida que la especificidad de los ordenamientos jurídicos lo permite; y v) deben buscar la armonía en términos de las necesidades de los diferentes actores, objetivos, planeación, gestión, ejecución, instrumentos jurídico-administrativos. Un consenso de todo esto, es complejo.

Dada la complejidad para convenir una acepción de política pública es relevante contar con las herramientas/instrumentos/paradigmas/ regímenes que permitan analizar y comparar los matices entre determinados ámbitos de los poderes públicos y su corresponsabilidad entre las acciones de gobierno y las demandas sociales. Es aquí donde los aspectos metodológicos cobran importancia. La enseñanza de la metodología y de los métodos conducen en dirección a un pensamiento analítico derivado de la observación científica.

\section{METODOLOGÍA}

El artículo se formula bajo el esquema de una investigación cualitativa, que habrá de hacer uso de teorías y métodos que habrán de ser soporte para el análisis y el tratamiento de las información. De ahí que, éste revista de un carácter documental dado que se apropia de "un procedimiento científico, un proceso sistemático de indagación, recolección, organización, análisis e interpretación de información o datos en torno a un determinado tema. Al igual que otros tipos de investigación, éste es conducente a la construcción de conocimientos" (Rizo, 2015, Unidad II).

La investigación cualitativa en su proceso introduce una sucesión de pasos, que inicia por acogerse a una técnica documental para después definir el marco teórico-metodológico que mucho ayudará al análisis y a la discusión para, 
finalmente, formular resultados/hallazgos sobre el objeto-sujeto de investigación (Baena, 2017).

La investigación cualitativa descansa en que su entramado hace uso de "técnicas específicas para la recolección, manejo y análisis de datos, buscando no causas generales sino explicaciones de actos dentro de un contexto y función específico" (Ocegueda, 2002, p. 44). Este artículo es una investigación cualitativa debido a que este paradigma proporciona la comprensión del objeto de estudio

De ahí que, lo primero que se hizo es la búsqueda de documentos posteriores al 2011, teniendo como base aquellos textos que refieren a los autores Altamirano y Martínez (2011). Posteriormente, de los resultados obtenidos, fueron seleccionados aquellos documentos de autores distintos a los criterios de búsqueda. Después, de lo obtenido se exploró cada documento a fin de corroborar e identificar la referencia y modo de emplear las reflexiones del texto de Altamirano y Martínez (2011) sobre el método comparado.

En este mismo apartado es pertinente señalar que en el campo de las ciencias sociales y en el ámbito metodológico, por el uso de la investigación se considera que este trabajo contribuye a la información y descripción del objeto de estudio, en este caso las políticas públicas y el método comparado. Por la temporalidad, la investigación es retrospectiva, toda vez que delimita la búsqueda a los textos que citan y refieren el texto de Altamirano y Martínez de 2011 al 2020.

Por el conocimiento disponible, la investigación es cualitativa (incluso descriptiva), puesto que se indican las características del objeto de estudio y se contribuye con información compendiosa y circunscrita al tema. Por lo que, como ya se afirmó es una investigación documental, debido a que permite citar documentos informativos respecto a las investigaciones realizadas en torno al objeto de estudio.

\section{DISCUSIÓN}

A mediados de los años 70, Anderson (1975) apuntaba que el análisis comparado en materia de políticas públicas era un campo inexistente. Sin embargo, el trabajo de Allison (1969), se puede decir, sentó las bases para comparar la hechura de políticas públicas — como en su momento lo definió Aguilar en su texto del mismo nombre (1972) — y, así, derivar acciones de gobierno. Más recientemente, Grau y Mateos (2002) indican que las políticas públicas comparadas constituyen un campo de estudio consolidado, toda vez que el objetivo es explicar las similitudes y diferencias, para hallar variables significativas y explicativas de los cursos de acción.

En este sentido, se puede observar la utilidad de conocer el método comparado, de poner en práctica la comparación a efecto de contar con un soporte y rigor metodológico en la construcción de políticas públicas; esto frente al "creciente popurrí de informaciones dispares, poco acumulables y probablemente engañosas" (Sartori, 2011, p. 42).

Para ejemplificar la relación entre el método comparado y las políticas públicas, se hace una revisión de diferentes textos que acuden al método comparado para analizar diferentes ámbitos de las políticas públicas, los cuales refieren algunas reflexiones o conclusiones expuestas en Altamirano y Martínez (2011).

De la búsqueda se eligieron doce textos publicados entre 2014 y 2020, siendo descartados aquellos publicados por los mismos autores de manera individual o conjunta; resultaron 12 textos en formato de artículo científico o tesis de posgrado (doctoral y maestría). Los trabajos emplean el método comparado para temas de innovación y tecnología, educación, procesos de paz. Al tiempo, también, refieren los casos en Brasil, Japón, Colombia, España y México. Suman 7 tesis doctorales, 2 tesis de maestría, y 3 artículos. 
Cuadro 1. Texto y objetivo de estudio de casos sobre uso del método comparado para el análisis de diferentes políticas públicas

\begin{tabular}{|c|c|c|c|c|}
\hline Texto & Objetivo del texto & Tipo & País & $\begin{array}{c}\text { Uso del método } \\
\text { comparado }\end{array}$ \\
\hline $\begin{array}{l}\text { Caballero S. (2020). Bricolaje institucional } \\
\text { y efectos en los medios de vida por } \\
\text { el Programa de Pago por Servicios } \\
\text { Ambientales Hidrológicos, en la Reserva de } \\
\text { la Biosfera de la Sepultura, Chiapas. Tesis } \\
\text { de Universidad Autónoma De Chiapas. }\end{array}$ & $\begin{array}{l}\text { Analizar el Programa de Pago } \\
\text { por Servicios Ambientales } \\
\text { (PSA), a partir de experiencias } \\
\text { de poblaciones de dos núcleos } \\
\text { agrarios de la Reserva de la } \\
\text { Biosfera la Sepultura, Chiapas. }\end{array}$ & $\begin{array}{c}\text { Tesis } \\
\text { doctoral }\end{array}$ & México & $\begin{array}{l}\text { Comparar las } \\
\text { experiencias } \\
\text { en el marco del } \\
\text { PSA }\end{array}$ \\
\hline $\begin{array}{l}\text { Silva, E. da; Valentim, M.; y La Mano, } \\
\text { M. (2020). "Avaliação de indicadores de } \\
\text { ciência, tecnologia e inovação do Brasil } \\
\text { e da Espanha: estudo comparativo", en } \\
\text { Capa, V. 26, N. 2, mayo/ago. }\end{array}$ & $\begin{array}{l}\text { Analizar indicadores de ciencia, } \\
\text { tecnología e innovación en } \\
\text { Brasil y España y su relación } \\
\text { con los procesos de producción. }\end{array}$ & Artículo & Brasil & $\begin{array}{l}\text { Compara } \\
\text { indicadores de } \\
\text { dos países }\end{array}$ \\
\hline $\begin{array}{l}\text { Silva, E. da; Hassem, A.; Capinzaiki, } \\
\text { S.; \& Mallavazi, V. (2020). "Strategies } \\
\text { in Innovation Policies: a comparative } \\
\text { study", European Public \& Social } \\
\text { Innovation Review, 5(1), 10-21. }\end{array}$ & $\begin{array}{l}\text { Analizar las diferencias y } \\
\text { similitudes entre las políticas } \\
\text { de desarrollo del Objetivo } 9 \text { de } \\
\text { la Agenda } 2030 \text { y la Estrategia } \\
\text { brasileña de Ciencia, Tecnología } \\
\text { e Innovación. }\end{array}$ & Artículo & España & $\begin{array}{l}\text { Compara } \\
\text { políticas en } \\
\text { materia de } \\
\text { innovación }\end{array}$ \\
\hline $\begin{array}{l}\text { López, R. J. (2019) Relación entre marco } \\
\text { institucional y política de gasto público: una } \\
\text { visión comparada entre Chile y Venezuela } \\
\text { (2000-2010). Tesis de la Universidad } \\
\text { Complutense de Madrid. }\end{array}$ & $\begin{array}{l}\text { Comparar, entre Chile y } \\
\text { Venezuela, las similitudes de los } \\
\text { ámbitos económico y político; } \\
\text { las diferencias de izquierda en el } \\
\text { plano político. }\end{array}$ & $\begin{array}{l}\text { Tesis } \\
\text { doctoral }\end{array}$ & España & $\begin{array}{l}\text { Compara } \\
\text { el marco } \\
\text { institucional de } \\
\text { dos países }\end{array}$ \\
\hline $\begin{array}{l}\text { Martínez, J. (2019). Los consejos escolares } \\
\text { de participación social (CEPS), ¿estrategia } \\
\text { para la autonomía en las escuelas primarias } \\
\text { indígenas y generales de la región V altos } \\
\text { tsotsil tseltal del estado de Chiapas? Tesis } \\
\text { de la Universidad Autónoma de Chiapas. }\end{array}$ & $\begin{array}{l}\text { Analizar y comparar } 25 \text { escuelas } \\
\text { con CEPS, en la Región V Altos } \\
\text { Tsotsil Tseltal, Chiapas. }\end{array}$ & $\begin{array}{l}\text { Tesis } \\
\text { doctoral }\end{array}$ & México & $\begin{array}{l}\text { Compara la } \\
\text { constitución } \\
\text { de consejos } \\
\text { escolares }\end{array}$ \\
\hline $\begin{array}{l}\text { Silva, E. da }(2018) \\
\text { O conhecimento científico no contexto de } \\
\text { sistemas nacionais de inovação: análise } \\
\text { de políticas públicas e indicadores de } \\
\text { inovação. Tese do Doutorado Programa de } \\
\text { PósGraduação em Ciência da Informação, } \\
\text { do Universida de Estadual Paulista. }\end{array}$ & $\begin{array}{l}\text { Investigar las políticas públicas } \\
\text { e institucionales, los indicadores } \\
\text { de innovación en Brasil y } \\
\text { España respecto a la producción } \\
\text { e intercambio de conocimiento } \\
\text { científico. }\end{array}$ & $\begin{array}{c}\text { Tesis } \\
\text { doctoral }\end{array}$ & Brasil & $\begin{array}{l}\text { Compara } \\
\text { políticas en } \\
\text { materia de } \\
\text { innovación de } \\
\text { dos países }\end{array}$ \\
\hline $\begin{array}{l}\text { Cortés, S. (2017). Colaboración } \\
\text { interinstitucional universitaria para la } \\
\text { construcción de un servicio educativo: } \\
\text { MOOC de alfabetización. Tesis de la } \\
\text { Benemérita Universidad Autónoma De } \\
\text { Puebla }\end{array}$ & $\begin{array}{l}\text { Documentar la experiencia de } \\
\text { trabajo de tres Instituciones de } \\
\text { Educación Superior del ámbito } \\
\text { público. }\end{array}$ & $\begin{array}{l}\text { Tesis de } \\
\text { maestría }\end{array}$ & México & $\begin{array}{l}\text { Compara tres } \\
\text { dimensiones } \\
\text { de análisis para } \\
\text { tres casos }\end{array}$ \\
\hline $\begin{array}{l}\text { Guerrero, L. (2017). "El estudio de } \\
\text { la subjetividad. Una Mirada desde la } \\
\text { educación comparada", In Crescendo. } \\
\text { Educación y Humanidades; 4(1): 50-71. }\end{array}$ & $\begin{array}{l}\text { Establecer una propuesta } \\
\text { para una aproximación a la } \\
\text { subjetividad desde la perspectiva } \\
\text { de la educación comparada. }\end{array}$ & Artículo & Perú & $\begin{array}{l}\text { Propone una } \\
\text { metodología } \\
\text { comparada para } \\
\text { el estudio de la } \\
\text { subjetividad en } \\
\text { el marco de la } \\
\text { educación. }\end{array}$ \\
\hline
\end{tabular}




\begin{tabular}{|c|c|c|c|c|}
\hline $\begin{array}{l}\text { Valencia, G. D. (2017). Organizarse } \\
\text { para negociar la paz. Un análisis } \\
\text { político transaccional de las estructuras } \\
\text { de gobernanza de la paz negociada } \\
\text { en Colombia, 1981-2016. Tesis de la } \\
\text { Universidad de Antioquia }\end{array}$ & $\begin{array}{l}\text { Analizar la dinámica y } \\
\text { evolución de organizaciones } \\
\text { e instituciones creadas por el } \\
\text { gobierno nacional para negociar } \\
\text { la paz en Colombia. }\end{array}$ & $\begin{array}{c}\text { Tesis } \\
\text { doctoral }\end{array}$ & Colombia & $\begin{array}{l}\text { Compara } \\
\text { los marcos } \\
\text { institucionales } \\
\text { de manera } \\
\text { trasversal }\end{array}$ \\
\hline $\begin{array}{l}\text { Sanoni, Ch,P. (2015), Politics of Regulation } \\
\text { of ICTs for Telemedicine: Analysis of Rural } \\
\text { Areas of Peru. Tesis de la Universidad de } \\
\text { Tsukuba, Japón. }\end{array}$ & $\begin{array}{l}\text { Reconocer los efectos de } \\
\text { la política nacional en la } \\
\text { formulación de políticas para las } \\
\text { TIC's en el Perú. }\end{array}$ & $\begin{array}{c}\text { Tesis } \\
\text { doctoral }\end{array}$ & Japón & $\begin{array}{l}\text { Compara la } \\
\text { implementación } \\
\text { de políticas en } \\
\text { tres regiones } \\
\text { rurales } \\
\end{array}$ \\
\hline $\begin{array}{l}\text { Ramírez, M. LM. (2015). Actitudes } \\
\text { Democráticas Para La Participación Social } \\
\text { En Jóvenes De Secundarias Generales En } \\
\text { Chiapas, Durango Y El Distrito Federal. } \\
\text { Tesis de la Universidad Iberoamericana }\end{array}$ & $\begin{array}{l}\text { Analizar las } r \text { actitudes } \\
\text { democráticas que presentan } \\
\text { los jóvenes de escuelas } \\
\text { secundarias generales y que son } \\
\text { la base para generar condiciones } \\
\text { participativas. }\end{array}$ & $\begin{array}{l}\text { Tesis de } \\
\text { maestría }\end{array}$ & México & $\begin{array}{l}\text { Compara padres } \\
\text { de familia, } \\
\text { estudiantes, } \\
\text { maestros y } \\
\text { directores en } \\
27 \text { escuelas, en } \\
3 \text { estados de } \\
\text { la República } \\
\text { mexicana: } \\
\text { Chiapas, } \\
\text { Durango y } \\
\text { Distrito Federal. }\end{array}$ \\
\hline $\begin{array}{l}\text { Ruiz, R. de los R. (2014). Conformación } \\
\text { de redes académicas en las Universidades } \\
\text { Públicas a partir de las políticas } \\
\text { educativas: Un análisis comparativo en } \\
\text { dos Universidades. Tesis de la Universidad } \\
\text { Autónoma del Estado de Hidalgo. }\end{array}$ & $\begin{array}{l}\text { Identificar los factores exógenos } \\
\text { y endógenos que favorecen u } \\
\text { obstaculizan la conformación } \\
\text { de redes académicas a través del } \\
\text { estudio de casos con perspectiva } \\
\text { comparada. }\end{array}$ & $\begin{array}{c}\text { Tesis } \\
\text { doctoral }\end{array}$ & México & $\begin{array}{l}\text { Compara dos } \\
\text { universidades } \\
\text { en el contexto } \\
\text { de redes de } \\
\text { colaboración. }\end{array}$ \\
\hline
\end{tabular}

Derivado del cuadro 1, se sintetiza lo siguiente:

1. Los diferentes ámbitos de las políticas públicas en los textos referidos no son limitativos en cuanto al uso del método comparado. Se puede aplicar para comparar políticas, instituciones, países, localidades, marcos normativos, etcétera.

2. Los textos referidos permiten observar que el método comparado da resultados puntuales con un número limitado de casos y con el manejo de un número de variables (también) limitado. Los casos analizan unidades de observación comparables entre países, regiones y políticas, esto en lugar de comparaciones sin propósitos claros, como el texto que compara padres de familia, estudiantes, maestros y directores en 27 escuelas, en tres estados.

3. El uso del método comparado y, particularmente, el estudio limitado de casos ofrece consistencia para generar axiomas puntuales respecto a las unidades de análisis.

4. Los textos referidos contribuyen a resaltar las diferencias y similitudes en las unidades de análisis, que bien pueden ser generales y/o particulares. Y derivado de ello sobresale el uso del comparativismo como marco metodológico.

5. El método comparado — según se observa en los textos referidos - permitió precisar, afirmar o descartar las visiones generales y/o particulares respecto a las unidades de análisis. Tal hecho hace valer lo señalado por Badie y Hermet (1993), quienes afirman que el método comparado “(...) tiene como principal beneficio desarticular las certidumbres construidas, con el fin de precisar, afirmar, corregir o enmendar los paradigmas, y con el tiempo, definir la frontera que separe los 
dominios de lo universal y de lo particular" (p. 8).

6. Se observó que en los textos referidos ponderan lo afirmado por Altamirano y Martínez (2011), en lo relativo a: i) uso limitado de casos, ii) establecer similitudes y diferencias con base en contextos o realidades similares; iii) superar los estudios descriptivos; iv) el valor de la sistematización de la información; y v) un solo caso estudio no constituye una base sólida para plantear generalizaciones. Esto permite considerar que el texto referido tiene una carga valorativa como herramienta que coadyuva al esclarecimiento del uso del método comparado en el trabajo de investigación y análisis de políticas públicas.

Este ejercicio sobre el uso del método comparado para el análisis de diferentes políticas permite no perder de vista que el estudio de casos está en función de la delimitación del objeto-sujeto de estudio y a la unidad de análisis.

\section{CONCLUSIONES}

En las ciencias sociales, un marco metodológico se trata de la delimitación reflexionada de un procedimiento para el estudio del objeto-sujeto de investigación, al tiempo, también, ofrece una trama para desarrollar un discurso explicativo sobre la razón del objeto de estudio. Una acepción restringida, como lo es un marco metodológico, no es limitativa de los métodos de estudio, ni de los resultados y alcances de los análisis.

El método comparado figura como marco metodológico para el tratamiento de los fenómenos que ocurren en la realidad social; en especial porque permite distinguir las diferencias y similitudes, así como sus variantes, considerando un determinado contexto en el que tienen lugar los casos de estudio.

El estudio limitado de casos ofrece consistencia para generar tipologías sobre casos similares en contextos equivalentes, a través de establecer una relación entre las variables, o bien, determinar el vínculo entre los casos. Sin embargo, el método comparado no es infalible, dado que tiene sus propios riesgos metodológicos, que deben ser contenidos a través de un arduo trabajo reflexivo sobre los casos y/o variables que pueden ser tan disimiles a efecto de la comparación no sea factible.

Toda unidad de análisis que se enmarcan en el método comparado deben cumplir con la cláusula ceteris paribus.

El método comparado muestra su valía como una herramienta de gran alcance en la elaboración e implementación de las políticas públicas ( $\mathrm{u}$ otros rubros), al permitir recobrar experiencias de otras latitudes a efecto de conocer bien a bien los elementos o componentes que se ciñen en su contexto.

\section{REFERENCIAS}

1. Aguilar, L. (1992). La hechura de las politicas, México: Miguel Ángel Porrúa.

2. Altamirano, M., y Martínez, A. (2011). El método comparado y el neo-institucionalismo como marco metodológico para la investigación en las Ciencias Sociales. Mundo Siglo XXI. Vol. VII (25), 55-63. México: CIECAS-IPN.

3. Álvares-Gayou, J. L. (2006). Cómo hacer investigación cualitativa. Fundamentos y metodología, México: Paidós.

4. Anderson, C. W. (1975). Systems and Strategy in Comparative Policy Analysis: A Plea for Contextual and Experiential Knowledge. En W.B. Gwyn and G.C. Edwards (eds.). Perspectives on Policy making. Tulane: Studies in Political Science.

5. Atria, R. (2005). Políticas sociales. Concepto y Diseño, en Estudios sociales 116, Semestre 2. Corporación de Promoción Universitaria. 
6. Badie, B. y Hermet, G. (eds.) (1993). Política comparada, México: FCE.

7. Baena, G. M. (2017). Metodología de la investigación (3a. ed.). Recuperado el 16 de abril de 2021, de: http://ebookcentral.proquest.com. Created from bibliotecacijsp on 2018-07-31 15:53:16.

8. Blondel, J. (1999). Then and now: comparative politics, en Political Studies, 47(1), 152-160.

9. Borges, R. (1995). El estudio de caso como instrumento pedagógico y de investigación en políticas públicas, Estudio de Caso 4. Magíster en Gestión y Políticas Públicas. Santiago de Chile: Universidad de Chile.

10. Bourdieu, P. (1975). El oficio del sociólogo, México: Siglo XXI.

11. Caïs, J. (1997). Metodología del análisis comparativo, no. 21, Madrid: Centro de Investigaciones Sociológicas (CIS).

12. Camou, A. (2001). Estudio preliminar, en Camou, A. (comp.) Los desafios de la gobernabilidad. Estudio preliminar y compilación, México: FLACSO/IIS-UNAM/Plaza y Valdés.

13. Cárdenas. J. (2017). Del estado absoluto al estado neoliberal. Universidad Nacional Autónoma de México-Instituto de Investigaciones Jurídicas. [fecha de Consulta 22 de Marzo de 2021]. Disponible en: https://archivos.juridicas.unam.mx/www/bjv/libros/9/4310/10.pdf.

14. Catalá, M. (1951). Concepto de política social. Cuadernos de Política Social 11: 21-46. [fecha de Consulta 18 de Marzo de 2021]. Disponible en: https://dialnet.unirioja.es/ejemplar/438282.

15. Collier, D. (1994). El método comparativo, dos décadas de cambios, en G. Sartori y L. Morlino (comps.), La comparación en las ciencias sociales, Madrid: Editorial Alianza.

16. Creswell, J. (2002). Research design: qualitative, quantitative and mixed method approaches, London: Sage.

17. De la Torre, R.; Rodríguez-Oreggia, E.; y Soloaga, I. (2018). Política social y bienestar: México desde el año 2000, México: CIDE.

18. DEJ-RAE (2017). Ordenamiento Jurídico. [fecha de Consulta 13 de enero de 2021]. Disponible en: http://dej.rae.es/\#/entry-id/E171690.

19. Dye, T. R. (1975) Understanding Public Policy, $12^{\text {th }}$ ed., New Jersey: Prentice-Hall.

20. Farge, C. (2007). El Estado de bienestar. Enfoques, XIX(1-2),45-54. [fecha de Consulta 21 de marzo de 2021]. Disponible en: https://www.redalyc.org/articulo.oa?id=259/25913121005.

21. Fernández, A. y Rozas, M. (1988). Políticas Sociales y Trabajo Social. Ed. Buenos Aires: HVMANITAS.

22. Fernández, P.; Bello, A.; y Messarini, P. (2016). Políticas públicas e instrumentos para el desarrollo de la cultura científica en América Latina, Montevideo: LATU/UNESCO.

23. González, M. (2000). Lo vigente y lo emergente: Tendencias recientes en la política social en México. Logros y retos: Una evaluación cualitativa de Progresa, en Más oportunidades para las familias pobres. Evaluación de Resultados del Programa de Educación, Salud y Alimentación (Impacto a nivel comunitario). México: Secretaria de Desarrollo Social.

24. Grau, M., y Mateos, A. (eds.) (2002). Análisis de politicas públicas en España: enfoques y casos. Valencia: Tirant lo Blanch.

25. Hall A., P., and Taylor C. R. (1998). The potential of historical institutionalism a response to Hay and Wincott, en Political Studies 46(5): 958-62.

26. Isuani, F. J. (2012). Instrumentos de políticas públicas. Factores clave de las capacidades estatales, en DAAPGE 12(19): 51-74, Santa Fe, Argentina.

27. Kauffer, E. (2002). Las políticas públicas: algunos apuntes generales, en Ecofronteras, (16), México: El Colegio de la Frontera Sur.

28. Khun, T. S. (1971). La estructura de las revoluciones cientificas. México: FCE.

29. Koelble, T. A. (1995), The New Institutionalism in Political Science and Sociology, en Comparative Politics 27(2): 231-243.

30. Lahera, E. (2004). Política y políticas públicas, en Serie Políticas Sociales 95. Santiago de Chile: CEPAL.

31. Lijphart, A. (1971). Comparative politics and the comparative method, en American Political Science Review, 65(3): 682- 693

32. Llamazares, I. (1995). El análisis comparado de los fenómenos políticos, en Revista de Estudios Políticos 89: 281-297.

33. Mackie, T., y Marsh, D. (1997). El método comparativo, en David Marsh y Gerry Stocker (eds.), Teoría y métodos de la ciencia política, Madrid: Editorial Alianza.

34. Macridis, C. R. (1981). Revisión del campo del estudio comparado de las formas de gobierno, en 
J. Blondel et al, El gobierno: estudios comparados, Madrid: Alianza, Madrid. pp. 51-58.

35. March, J. G. y Olsen, J. P. (1984). The new institutionalism: organizational factor in political life, en American Political Science Review, 78 (3): 734-749.

36. Mèny, I y Thoënig, J. C. (1992). Las políticas públicas, Barcelona: Ariel.

37. Merriam, S. B. (1988). Case studies as Qualitative research. En Qualitative research and Case study applications in education. Revised and expanded from Case study research in education. [fecha de Consulta 1 de febrero de 2021]. Disponible en: http://eds-courses.ucsd.edu/tep288a/ shortbook.pdf.

38. Morales, V. (2002), "On methodology as a science and the scientific method: a controversial territory”, en Revista de Pedagogía, 23(66), 121-146.

39. North, D. C. (1993). Instituciones, cambio institucional y desempeño económico, México: FCE.

40. Ocegueda, C. (2002). Metodología de la investigación. Métodos, técnicas y estructuración de trabajos académicos. México: s/e. [fecha de Consulta 16 de abril de 2021]. Disponible en: : https:// www.researchgate.net/publication/330409452_Metodologia_de la_Investigacion_Metodos Tecnicas y estructuracion_de trabajos_academicos.

41. Oszlak, O., \& O’Donnell, G. (1995). Estado y políticas estatales en América Latina: hacia una estrategia de investigación. Redes, 2(4),99-128.[fecha de Consulta 22 de Marzo de 2021]. Disponible en: https://www.redalyc.org/articulo.oa?id=90711285004.

42. Przeworski, A. (1987), Methods of cross-national. Research, 1970-1983: an overview, en M. Dierkes et. al., (eds.), Comparative policy research: learning from experience, Berlin: Aldershot.

43. Rizo, J. (2015). Técnicas de investigación documental. Nicaragua: Universidad Nacional Autónoma de Nicaragua, Managua Facultad Regional Multidisciplinaria de Matagalpa UNANFAREM-Matagalpa. [fecha de Consulta 16 de abril de 2021]. Disponible en: https://repositorio. unan.edu.ni/12168/1/100795.pdf.

44. Rose, R. (1991), "Comparing form of comparative analysis", en Political Science, 39(3): 446-462.

45. Sartori, G. (1994). Comparación y método comparativo. en G. Sartori y L. Morlino (comps.), La comparación en las ciencias sociales, Madrid: Editorial Alianza..

46. Sartori, G. (2011), Cómo hacer ciencia política: lógica, método y lenguaje en las ciencias sociales, Madrid: Taurus.

47. Silva, F., Martínez, G., Altamirano, M., y Lara, I. (2020). "Tecnología de la información y comunicación en los posgrados PNPC", 3C TIC. Cuadernos de Desarrollo Aplicados a las TIC, 9(3), 95-105.

48. Thoënig, J. C. (2006). El rescate de las publicness en los estudios de la organización, en Gestión y Política Pública 15 (2): 229-258.

49. Wiarda, H. (1991), Comparative politics: past and present, en Howard J. Wiarda, (ed.), New directions in comparative politics (Boulder, Westview), USA: Westview Press.

50. Wright, V., Hague, R., Harrop, M., and Breslin, S. (eds.) (1992), Comparative government and politics: an introduction, England: The MacMillan Press. 